\title{
Диэлектрические свойства и электропроводность легированного серебром монокристалла $\mathrm{TIGaS}_{2}$
}

\author{
(C) С.Н. Мустафраева ${ }^{1}$, С.М. Асадов ${ }^{2}$, Э.М. Керимова ${ }^{1}$ \\ ${ }^{1}$ Институт физики Национальной академии наук Азербайджана, \\ Az-1143 Баку, Азербайджан \\ ${ }^{2}$ Институт катализа и неорганической химии Национальной академии наук Азербайджана, \\ Az-1143 Баку, Азербайджан \\ E-mail: solmust@gmail.com
}

(Получена 17 января 2017 г. Принята к печати 26 июня 2017 г.)

Исследовано влияние ионов серебра (2мол\%) на диэлектрические свойства и электропроводность выращенных методом Бриджмена-Стокбаргера монокристаллов $\mathrm{TlGaS}_{2}$. Экспериментальные результаты по изучению частотной дисперсии диэлектрических коэффициентов монокристаллов $\mathrm{TlGaS}_{2}\langle 2 \mathrm{moл \%} \mathrm{Ag}\rangle$ позволили установить природу диэлектрических потерь, механизм переноса заряда, оценить плотность состояний вблизи уровня Ферми, их разброс, среднее время и среднюю длину прыжков, а также концентрацию глубоких ловушек, ответственных за проводимость на переменном токе. Легирование монокристалла $\mathrm{TlGaS}_{2}$ серебром приводило к увеличению плотности состояний вблизи уровня Ферми и уменьшению среднего времени и длины прыжков.

DOI: $10.21883 /$ FTP.2018.02.45438.8517

\section{1. Введение}

Путем легирования полупроводников различными примесями, как известно, можно существенно изменять их физические характеристики. Слоистые монокристаллы $\mathrm{TlGaS}_{2}$ и твердые растворы на его основе привлекают внимание исследователей своими важными физическими и физико-химическими свойствами. В настоящее время изучены область существования, кристаллическая структура, электрические и диэлектрические свойства легированных кристаллов на основе $\mathrm{TlGaS}_{2}[1-8]$. Изучение электрических свойств нелегированных монокристаллов $\mathrm{TlGaS}_{2}$ на постоянном $(d c)$ [1] и переменном $(a c)$ токе [2] показало, что при температурах $T<200 \mathrm{~K}$ и частотах $f=5 \cdot 10^{4}-10^{6}$ Гц в них имеет место прыжковая $d c$ - и $a c$-проводимость по локализованным вблизи уровня Ферми состояниям. Было показано, что результаты изучения $d c$ - и $a c$-проводимости кристаллов $\mathrm{TlGaS}_{2}$, взятых из одной технологической партии, хорошо согласуются друг с другом. В силу своей слоистости структуры монокристаллы $\mathrm{TlGaS}_{2}$ склонны к политипизму, поэтому физические параметры этих кристаллов, взятых из разных партий, не всегда согласуются друг с другом.

В работах [2-8] были изучены диэлектрические свойства как нелегированных монокристаллов $\mathrm{TlGaS}_{2}$ [2], так и легированных ионами переходных и редкоземельных элементов, в частности хромом [3], марганцем [4], кобальтом [5], эрбием [6], иттербием [7,8]. Было показано, что легирование $\mathrm{TlGaS}_{2}$ приводит к существенному изменению диэлектрических коэффициентов полученных монокристаллов и изменяет в них природу диэлектрических потерь. Из известной фазовой диаграммы системы $\mathrm{Tl}_{2} \mathrm{~S}-\mathrm{Ga}_{2} \mathrm{~S}_{3}$ следует, что концентрационная область гомогенности на основе $\mathrm{TlGaS}_{2}$ является двухсторонней и составляет > 10 мол\%.
В настоящей работе приведены результаты изучения влияния серебра (2 мол\% Ag) на диэлектрические свойства и электропроводность полученных монокристаллов на основе $\mathrm{TlGaS}_{2}$, измеренные на переменном токе. Цель исследования состояла в том, чтобы выяснить механизмы проводимости, которые могут реализоваться в однофазных образцах на основе $\mathrm{TlGaS}_{2}$.

\section{2. Методика эксперимента}

Для решения поставленной задачи методом прямого синтеза были получены образцы сульфидов $\mathrm{TlGaS}_{2}$ и $\mathrm{TlGaS}_{2}\langle 2$ мол\% Ag $\rangle$. При этом исходными компонентами служили особо чистые химические элементы: Тl (Тл 00), $\mathrm{Ga}(\mathrm{Ga} 5 \mathrm{~N})$ и $\mathrm{S}$ (ос. ч. 16-5). Оценка растворимости серебра $(\mathrm{Ag})$ в кристаллической решетке $\mathrm{TlGaS}_{2}$ с учетом известных эффективных ионных радиусов элементов указывает на то, что радиус $\mathrm{Ag}^{+}(1.15 \AA)$ ближе к радиусу $\mathrm{Tl}^{+}(1.5 \AA)$, чем к радиусу $\mathrm{Ga}^{3+}(0.62 \AA)$, т.е. частичное замещение таллия серебром в слоистых кристаллах $\mathrm{TlGaS}_{2}$ более вероятно и соответствует условию образования раствора замещения.

Образцы $\mathrm{TlGaS}_{2}$ и $\mathrm{TlGaS}_{2}\langle 2$ мол\% Ag $\rangle$ синтезировали из взятых в стехиометрических соотношениях элементов путем непосредственного их сплавления в вакуумированных до $10^{-3}$ Па кварцевых ампулах при $(1000 \pm 3 \mathrm{~K}$ в течение 5-7 ч. С целью гомогенизации образцов их отжигали в вакууме при $750 \mathrm{~K}$ в течение 120 ч. Отожженные сплавы охлаждали до комнатной температуры в режиме выключенной печи. Завершенность синтеза и гомогенность полученных образцов, а также их индивидуальность контролировали методами дифференциального термического анализа (ДТА) и рентгеновского фазового анализа (РФА). Рентгенофазовый анализ сплавов проводился на дифрактометре ДРОН-2 
с использованием излучения $\mathrm{Cu} K_{\alpha}$ при комнатной температуре.

Из заранее синтезированных поликристаллов $\mathrm{TlGaS}_{2}$ и $\mathrm{TlGaS}_{2}\langle 2$ мол\%Ag $\rangle$ методом Бриджмена-Стокбаргера путем направленной кристаллизации выращивали монокристаллы. Для этого синтезированный поликристалл измельчали и помещали в кварцевую ампулу длиной 8-10 см с заостренным концом и внутренним диаметром 1 см. Вакуумированную до давления $10^{-3}$ Па кварцевую ампулу с поликристаллическим веществом помещали в двухтемпературную печь для выращивания монокристалла. В верхней зоне печи поддерживалась температура $(1170 \pm 3) \mathrm{K}$ (т.е. выше температуры плавления $\mathrm{TlGaS}_{2}$, которая составляет $1165 \mathrm{~K}$ ), а в нижней зоне - $(1110 \pm 3) \mathrm{K}$. Оптимальная скорость перемещения ампулы в печи равнялась $0.3-0.5 \mathrm{~cm} /$ ч, а градиент температуры у фронта кристаллизации составлял $(25 \pm 3) \mathrm{K}$.

Диэлектрические коэффициенты (действительная часть диэлектрической проницаемости $\varepsilon^{\prime}$ и тангенс угла диэлектрических потерь $\operatorname{tg} \delta$ ) монокристаллов $\mathrm{TlGaS}_{2}$ и $\mathrm{TlGaS}_{2}\langle 2$ мол\%Ag $\rangle$ были измерены резонансным методом [9]. Диапазон частот переменного электрического поля составлял $5 \cdot 10^{4}-3.5 \cdot 10^{7}$ Гц. Монокристаллические образцы из $\mathrm{TlGaS}_{2}$ и $\mathrm{TlGaS}_{2}\langle 2$ мол\% $\mathrm{Ag}\rangle$ для электрических измерений были изготовлены в виде плоских конденсаторов, плоскость которых была перпендикулярна кристаллографической $C$-оси образцов. В качестве электродов использована серебряная паста. Толщина образцов составляла $0.04 \mathrm{~cm}$, а площадь обкладок - $0.12 \mathrm{~cm}^{2}$. Все диэлектрические измерения проведены при $300 \mathrm{~K}$. Воспроизводимость положения резонанса составляла по емкости \pm 0.2 п, а по добротности $\pm(1.0-1.5)$ деления шкалы (добротность есть $Q=1 / \operatorname{tg} \delta)$. При этом наибольшие отклонения от средних значений составляли $3-4 \%$ для $\varepsilon^{\prime}$ и $7 \%$ для $\operatorname{tg} \delta$.

\section{3. Результаты и их обсуждение}

Анализ результатов РФА поли- и монокристаллических образцов на основе $\mathrm{TlGaS}_{2}$ показал, что полученные нами образцы, в которых концентрация серебра была 2 мол\%, являлись однофазными. Параметры решетки элементарной ячейки моноклинной сингонии (пространственная группа $\left.C_{2 h}^{6} C 2 / m\right) \mathrm{TlGaS}_{2}$ имеют значения $a=10.299 \AA, b=10.284 \AA, c=15.175 \AA, \beta=99.603^{\circ}$, которые хорошо согласуются с данными $[8,10]$. Эти параметры в пределах погрешности мало зависят от концентрации легирующего серебра; так, например, для изученного образца $\mathrm{TlGaS}_{2}\langle 2$ мол\% $\mathrm{Ag}\rangle c=(15.208 \pm 0.002) \AA$. На дифрактограммах помимо фазы $\mathrm{TlGaS}_{2}$ не обнаружены дополнительные рентгеновские максимумы.

На рис. 1 приведены частотные зависимости действительной составляющей комплексной диэлектрической проницаемости $\left(\varepsilon^{\prime}\right)$ образцов $\mathrm{TlGaS}_{2}$ и $\mathrm{TlGaS}_{2}$ $\langle 2$ мол\% $\mathrm{Ag}\rangle$. Видно, что в $\mathrm{TlGaS}_{2}$ (кривая 1) во

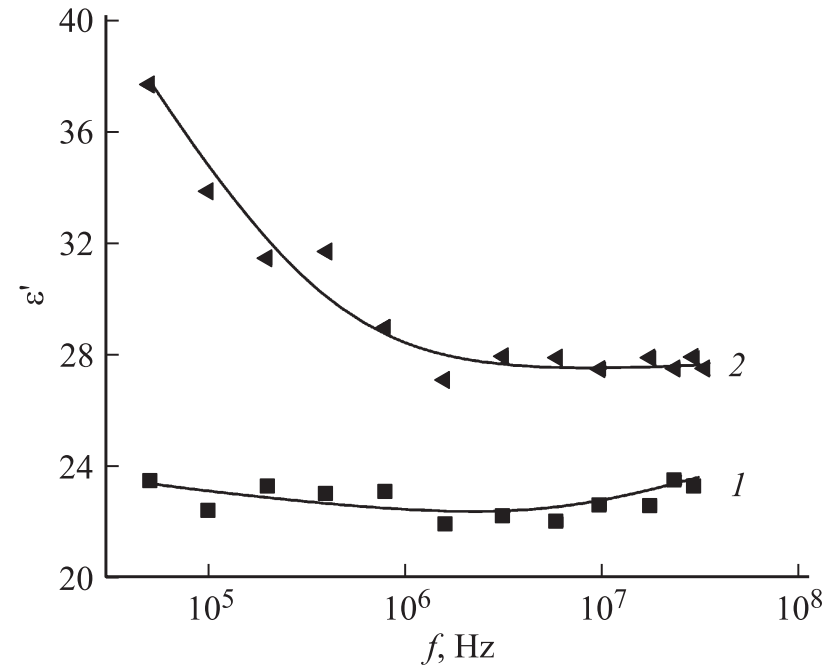

Рис. 1. Дисперсионные кривые $\varepsilon^{\prime}(f)$ для монокристаллов $\mathrm{TlGaS}_{2}$ (1) и $\mathrm{TlGaS}_{2}\langle 2$ мол\% Ag $\rangle$ (2) при $300 \mathrm{~K}$.

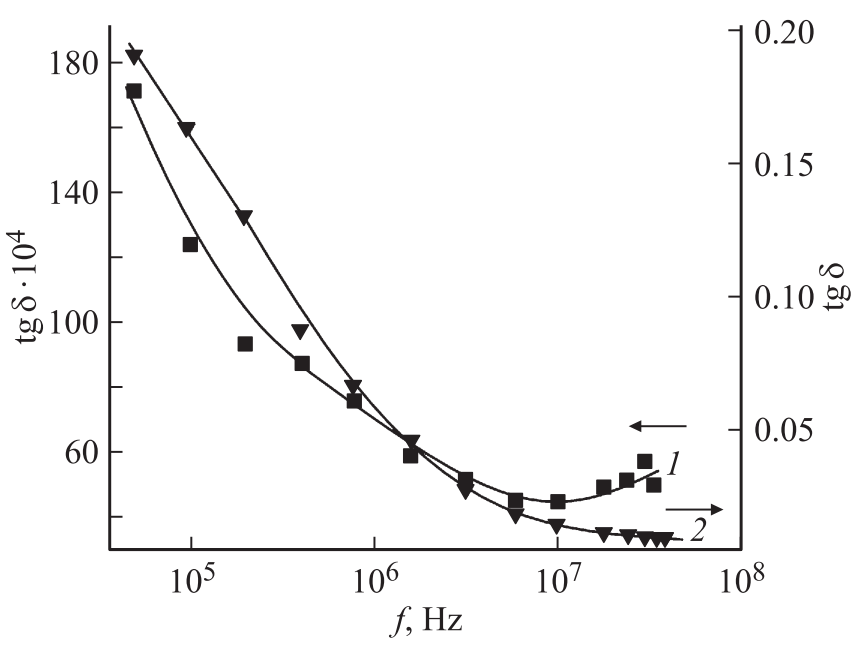

Рис. 2. Зависимости тангенса угла диэлектрических потерь $(\operatorname{tg} \delta)$ в монокристаллах $\mathrm{TlGaS}_{2}(1)$ и $\mathrm{TlGaS}_{2}\langle 2$ мол\% $\mathrm{Ag}\rangle$ (2) от частоты приложенного электрического поля.

всем изученном диапазоне частот существенной дисперсии $\varepsilon^{\prime}$ не наблюдается. Легирование кристалла $\mathrm{TlGaS}_{2}$ серебром приводит к заметной дисперсии $\varepsilon^{\prime}$ (кривая 2). Так, в $\mathrm{TlGaS}_{2}\langle 2$ мол\%Ag с изменением частоты от $5 \cdot 10^{4}$ до $3.5 \cdot 10^{7}$ Гц значение $\varepsilon^{\prime}$ уменьшалось от 37.7 до 28. Наблюдаемое в экспериментах монотонное уменьшение диэлектрической проницаемости монокристалла $\mathrm{TlGaS}_{2}\langle 2$ мол\%Ag с ростом частоты свидетельствует о релаксационной дисперсии [11]. Легирование серебром приводило к заметному увеличению $\varepsilon^{\prime}$ в $\mathrm{TlGaS}_{2}$. Так, при $f=5 \cdot 10^{4}$ Гц значение $\varepsilon^{\prime} \mathrm{TlGaS}_{2}\langle 2$ мол\% $\mathrm{Ag}\rangle$ более чем в полтора раза превышало значение $\varepsilon^{\prime} \mathrm{TlGaS}_{2}$.

Значения тангенса угла диэлектрических потерь $(\operatorname{tg} \delta)$ изученных монокристаллов $\mathrm{TlGaS}_{2}\langle 2$ мол\% $\mathrm{Ag}\rangle$ существенно превышали значения $\operatorname{tg} \delta$ в $\mathrm{TlGaS}_{2}$ (рис. 2). Гиперболический спад $\operatorname{tg} \delta$ с увеличением частоты в 


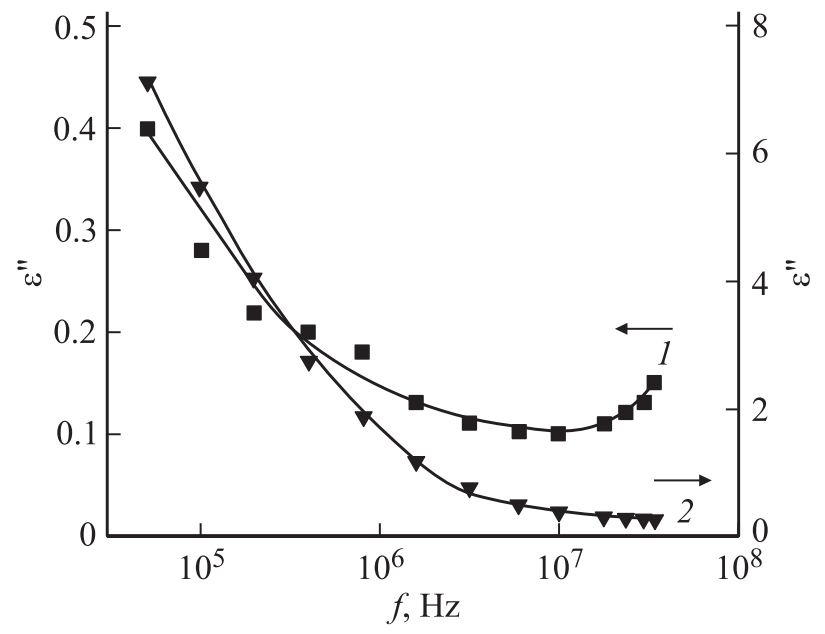

Pис. 3. Частотные зависимости мнимой составляющей комплексной диэлектрической проницаемости монокристаллов $\mathrm{TlGaS}_{2}$ (1) и $\mathrm{TlGaS}_{2}\langle 2$ мол\% Ag $\rangle$ (2).

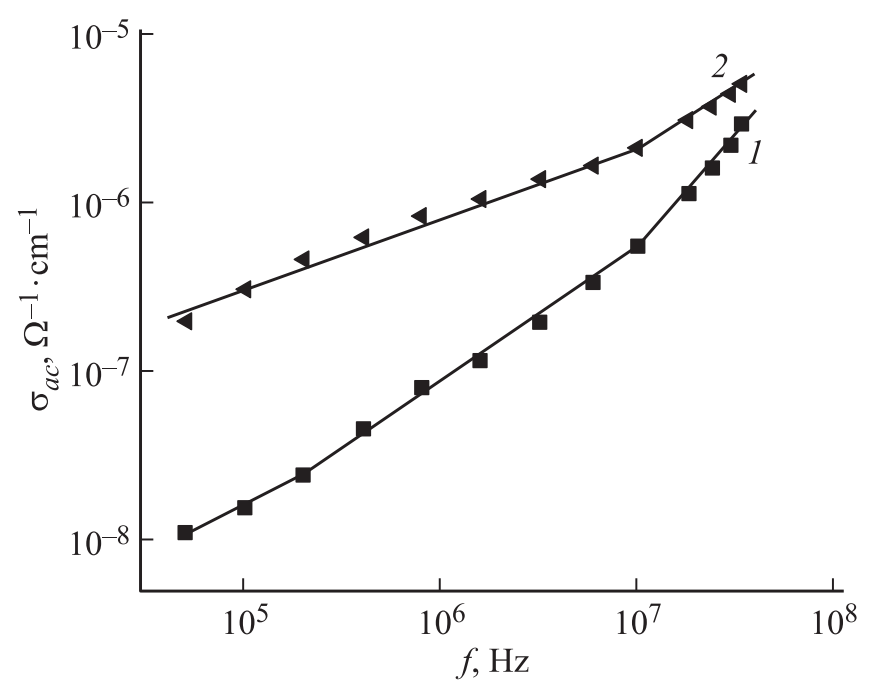

Рис. 4. Частотно-зависимая проводимость монокристаллов $\mathrm{TlGaS}_{2}(1)$ и $\mathrm{TlGaS}_{2}\langle 2$ мол\% $\mathrm{Ag}\rangle$ (2) при $T=300 \mathrm{~K}$.

монокристаллах $\mathrm{TlGaS}_{2}$ и $\mathrm{TlGaS}_{2}\langle 2$ мол\% $\mathrm{Ag}\rangle$ свидетельствует о потерях сквозной проводимости [11].

На рис. 3 приведены частотные зависимости мнимой части комплексной диэлектрической проницаемости $\left(\varepsilon^{\prime \prime}\right)$ монокристаллов $\mathrm{TlGaS}_{2}$ (кривая 1) и $\mathrm{TlGaS}_{2}\langle 2$ мол\% $\mathrm{Ag}\rangle$ (кривая 2). В отличие от монокристалла $\mathrm{TlGaS}_{2}$, в $\mathrm{TlGaS}_{2}\langle 2$ мол\% $\mathrm{Ag}\rangle$ дисперсионная кривая $\varepsilon^{\prime \prime}(f)$ характеризовалась довольно ощутимым спадом во всей изученной области частот. Так, если при увеличении частоты от $5 \cdot 10^{4}$ до $3.5 \cdot 10^{7}$ Гц значение $\varepsilon^{\prime \prime}$ $\mathrm{TlGaS}_{2}$ уменьшалось в 2.7 раз, то в $\mathrm{TlGaS}_{2}\langle 2$ мол\% $\mathrm{Ag}\rangle$ оно уменьшалось в 28 раз. При $f=5 \cdot 10^{4} \Gamma_{ц}$ значение $\varepsilon^{\prime \prime}$ монокристалла $\mathrm{TlGaS}_{2}\langle 2$ мол\% $\mathrm{Ag}\rangle$ в 18 раз превышало значение $\varepsilon^{\prime \prime}$ монокристалла $\mathrm{TlGaS}_{2}$.

На рис. 4 представлены результаты изучения частотно-зависимой $a c$-проводимости $\left(\sigma_{a c}\right)$ монокристал- лов $\mathrm{TlGaS}_{2}$ (кривая 1) и $\mathrm{TlGaS}_{2}\langle 2$ мол\% $\mathrm{Ag}\rangle$ (кривая 2) при $300 \mathrm{~K}$. В частотной области $5 \cdot 10^{4}-2 \cdot 10^{5}$ Гц $a c$-проводимость монокристалла $\mathrm{TlGaS}_{2}$ изменялась по закону $\sigma_{a c} \propto f^{0.6}$, а при $f=2 \cdot 10^{5}-2 \cdot 10^{7} \Gamma_{ц} \sigma_{a c} \propto f^{0.8}$. При $f>2 \cdot 10^{7}$ Гц имела место квадратичная зависимость $\sigma_{a c} \propto f^{2}$. Дисперсионная кривая $\sigma_{a c}(f)$ образца $\mathrm{TlGaS}_{2}\langle 2$ мол\% $\mathrm{Ag}\rangle$ имела два наклона:

$$
\sigma_{a c}=\sigma_{1}+\sigma_{2},
$$

где $\sigma_{1} \propto f^{0.4}$ в интервале частот $f=5 \cdot 10^{4}-10^{7} \Gamma ц$ и $\sigma_{2} \propto f^{0.8}$ при $f=10^{7}-3.5 \cdot 10^{7}$ Гц.

Проводимость на переменном токе ( $а$-проводимость) зонного типа, как известно, является в основном частотно-независимой вплоть до $10^{10}-10^{11}$ Гц. Наблюдаемая нами экспериментальная зависимость $\sigma_{a c} \propto f^{0.8}$ в кристаллах $\mathrm{TlGaS}_{2}$ свидетельствует о том, что она обусловлена прыжками носителей заряда между локализованными в запрещенной зоне состояниями. Это могут быть локализованные вблизи краев разрешенных зон состояния или локализованные вблизи уровня Ферми состояния [12,13]. Однако, так как в экспериментальных условиях проводимость по состояниям вблизи уровня Ферми всегда доминирует над проводимостью по состояниям вблизи краев разрешенных зон, полученный нами закон $\sigma_{a c} \propto f^{0.8}$ свидетельствует о прыжковом механизме переноса заряда по состояниям, расположенным в окрестности уровня Ферми. Предложенная в [14] формула для прыжковой проводимости имеет вид

$$
\sigma_{a c}(f)=\frac{\pi^{3}}{96} e^{2} k T N_{\mathrm{F}}^{2} r^{5} f\left[\ln \left(\frac{v_{\mathrm{ph}}}{f}\right)\right]^{4}
$$

где $e-$ заряд электрона, $k-$ постоянная Больцмана, $N_{\mathrm{F}}-$ плотность состояний вблизи уровня Ферми, $r=1 / \alpha-$ радиус локализации, $\alpha-$ постоянная спада волновой функции локализованного носителя заряда, $\psi \propto e^{-\alpha r}, v_{\mathrm{ph}}-$ фононная частота.

Согласно формуле (2), ac-проводимость зависит от частоты как $f\left[\ln \left(v_{\mathrm{ph}} / f\right)\right]^{4}$, т. е. при $f \ll v_{\mathrm{ph}} \sigma_{a c} \propto f^{0.8}$. Используя формулу (2), по экспериментально найденным значениям $\sigma_{a c}(f)$ образцов $\mathrm{TlGaS}_{2}$ и $\mathrm{TlGaS}_{2}\langle 2$ мол\% $\mathrm{Ag}\rangle$ вычислили плотность состояний на уровне Ферми. Вычисленные значения $N_{\mathrm{F}}$ для $\mathrm{TlGaS}_{2}$ и $\mathrm{TlGaS}_{2}\langle 2$ мол\% $\mathrm{Ag}\rangle$ составляли $5.9 \cdot 10^{18}$ и $1.3 \cdot 10^{19}{ }^{{ }^{2}} \mathrm{~B}^{1} \cdot \mathrm{cm}^{-3}$ соответственно, т.е. легирование монокристалла $\mathrm{TlGaS}_{2}$ серебром приводило к двукратному увеличению плотности состояний вблизи уровня Ферми. При вычислениях $N_{\mathrm{F}}$ для радиуса локализации взято значение $r=14 \AA$ [2], а $v_{\mathrm{ph}}$ для $\mathrm{TlGaS}_{2}$ составляет величину $\sim 10^{12} \Gamma_{ц}[15]$.

Согласно теории прыжковой проводимости на переменном токе средняя длина прыжков $(R)$ определяется по формуле [12]

$$
R=\frac{1}{2 \alpha} \ln \left(\frac{v_{\mathrm{ph}}}{f}\right)
$$

В формуле (3) значение $f$ соответствует средней частоте, при которой наблюдается закон $f^{0.8}$. Вычисленные по формуле (3) значения $R$ для монокристаллов $\mathrm{TlGaS}_{2}$ и 
$\mathrm{TlGaS}_{2}\langle 2$ мол\% $\mathrm{Ag}\rangle$ составляли 81 и $76 \AA$ соответственно. Эти значения $R$ в $\sim 6$ раз превышают среднее расстояние между центрами локализации носителей заряда в монокристаллах $\mathrm{TlGaS}_{2}$ и $\mathrm{TlGaS}_{2}\langle 2$ мол\% $\mathrm{Ag}\rangle$.

Значение $R$ позволило по формуле

$$
\tau^{-1}=v_{\mathrm{ph}} \exp (-2 \alpha R)
$$

определить среднее время прыжков в монокристаллах $\mathrm{TlGaS}_{2}$ и $\mathrm{TlGaS}_{2}\langle 2$ мол\% $\mathrm{Ag}\rangle: 9.9 \cdot 10^{-8}$ и $4.4 \cdot 10^{-8} \mathrm{c}$ соответственно.

По формуле

$$
\Delta E=\frac{3}{2 \pi R^{3} N_{\mathrm{F}}}
$$

в $\mathrm{TlGaS}_{2}\langle 2$ мол\% $\mathrm{Ag}\rangle$ оценен разброс локализованных вблизи уровня Ферми состояний $-\Delta E=8.4 \cdot 10^{-2}$ эВ, а по формуле

$$
N_{t}=N_{\mathrm{F}} \Delta E
$$

определена концентрация глубоких ловушек, ответственных за $а c$-проводимость $-N_{t}=1.1 \cdot 10^{18} \mathrm{~cm}^{-3}$ (в монокристалле $\mathrm{TlGaS}_{2}$ значение $N_{t}$ составляло $\left.8.8 \cdot 10^{17} \mathrm{~cm}^{-3}\right)$. Из полученных результатов следует, что легирование монокристалла $\mathrm{TlGaS}_{2}$ серебром приводило к существенному изменению диэлектрических коэффициентов полученных монокристаллов, увеличению плотности состояний вблизи уровня Ферми и уменьшению среднего времени и длины прыжков.

\section{4. Заключение}

В полученных монокристаллических образцах $\mathrm{TlGaS}_{2}$ и $\mathrm{TlGaS}_{2}\langle 2$ мол\% $\mathrm{Ag}\rangle$ изучена частотная дисперсия тангенса угла диэлектрических потерь $(\operatorname{tg} \delta)$, действительной $\left(\varepsilon^{\prime}\right)$ и мнимой $\left(\varepsilon^{\prime \prime}\right)$ составляющих комплексной диэлектрической проницаемости, а также $a c$-проводимости $\left(\sigma_{a c}\right)$ поперек слоев в области частот $f=5 \cdot 10^{4}-3.5 \cdot 10^{7}$ Гц. Установлено, что в $\mathrm{TlGaS}_{2}$ и $\mathrm{TlGaS}_{2}\langle 2$ мол\% $\mathrm{Ag}\rangle$ имеет место релаксационная дисперсия. Легирование монокристаллов $\mathrm{TlGaS}_{2}$ серебром приводит к модифицированию дисперсионных кривых $\varepsilon^{\prime}(f)$ и $\varepsilon^{\prime \prime}(f)$. Во всей изученной области частот в $\mathrm{TlGaS}_{2}$ и $\mathrm{TlGaS}_{2}\langle 2$ мол\% $\mathrm{Ag}\rangle$ имеют место потери на электропроводность. При высоких частотах $a c$-проводимость монокристаллов $\mathrm{TlGaS}_{2}$ и $\mathrm{TlGaS}_{2}\langle 2$ мол\% Ag $\rangle$ подчинялась закономерности $\sigma_{a c} \propto f^{0.8}$, характерной для прыжкового механизма переноса заряда по локализованным вблизи уровня Ферми состояниям. Оценены плотность $\left(N_{\mathrm{F}}\right)$ и разброс $(\Delta E)$ состояний, лежащих в окрестности уровня Ферми $N_{\mathrm{F}}=5.9 \cdot 10^{18}$ (для $\mathrm{TlGaS}_{2}$ ), $1.3 \cdot 10^{19}$ эВ $^{-1} \cdot \mathrm{cm}^{-3}$ (для $\mathrm{TlGaS}_{2}\langle 2$ мол\% $\left.\mathrm{Ag}\rangle\right), \Delta E=84-100$ мэВ. Среднее время составляет $\tau=9.9 \cdot 10^{-8} \mathrm{c}\left(\mathrm{TlGaS}_{2}\right), 4.4 \cdot 10^{-8} \mathrm{c}$ $\left(\mathrm{TlGaS}_{2}\langle 2\right.$ мол\% $\left.\mathrm{Ag}\rangle\right)$, длина прыжков $R=81 \AA$ (для $\mathrm{TlGaS}_{2}$ ) и $76 \AA$ (для $\mathrm{TlGaS}_{2}\langle 2$ мол\% $\mathrm{Ag}\rangle$ ). Таким образом, за счет легирования монокристалла $\mathrm{TlGaS}_{2}$ серебром можно управлять его диэлектрическими свойствами и $a c$-проводимостью.

\section{Список литературы}

[1] С.Н. Мустафаева, В.А. Алиев, М.М. Асадов. ФТТ, 40 (4), 612 (1998)

[2] С.Н. Мустафаева. ФТТ, 46 (6), 979 (2004).

[3] С.Н. Мустафаева. Журнал радиоэлектроники, № 8, 1 (2008).

[4] С.Н. Мустафаева. Изв. РАН. Неорг. матер., 42 (5), 530 (2006).

[5] С.Н. Мустафаева. Журнал радиоэлектроники, № 4, 1 (2009).

[6] С.Н. Мустафаева, М.М. Асадов, Э.М. Керимова, Н.3. Гасанов. Неорг. матер., 49 (12), 1271 (2013).

[7] В.Г. Гуртовой, А.У. Шелег, С.Н. Мустафаева, Э.М. Керимова. Изв. НАНБ. Сер. физ.-мат. наук, № 2, 98 (2015).

[8] А.У. Шелег, В.Г. Гуртовой, В.А. Чумак, С.Н. Мустафаева, Э.М. Керимова. Вестн. Гродненского гос. ун-та им. Я. Купалы. Сер. 2. Математика. Физика. Информатика, вычислительная техника и управление, 186 (1), 43 (2015).

[9] С.Н. Мустафаева. Все материаль. Энциклопедический справочник, № 10, 74 (2016).

[10] G.E Delgado, A.J. Mora, F.V. Perez, J. Gonzalez. Physica B, 391, 385 (2007).

[11] В.В. Пасынков, В.С. Сорокин. Материалы электронной техники (СПб-М.-Краснодар, 2004).

[12] Н. Мотт, Э. Дэвис. Электронные процессы в некристаллических веществах, пер. с англ. (М., Мир, 1974).

[13] С.Н. Мустафаева, С.М. Асадов. ФТП, 50 (9), 1159 (2016).

[14] M. Pollak. Phil. Mag., 23, 519 (1971).

[15] К.Р. Аллахвердиев, Е.А. Виноградов, Р.Х. Нани и др. В кн.: Физические свойства сложных полупроводников (Баку, Элм, 1982. с. 55).

Редактор Л.В. Шаронова

\section{Dielectric properties and conductivity of a Ag-doped $\mathrm{TIGaS}_{2}$ single crystal}

\author{
S.N. Mustafaeva ${ }^{1}$, S.M. Asadov' ${ }^{2}$ E.M. Kerimova ${ }^{1}$ \\ ${ }^{1}$ Institute of Physics, \\ Azerbaijan National Academy of Sciences, \\ Az-1143 Baku, Azerbaijan \\ ${ }^{2}$ Institute of Catalysis and Inorganic Chemistry, \\ Azerbaijan National Academy of Sciences, \\ Az-1143 Baku, Azerbaijan
}

\begin{abstract}
Influence of Ag-ions $(2 \mathrm{~mol} \% \mathrm{Ag})$ on dielectric properties and conductivity of grown by Bridgmen-Stockbarger method $\mathrm{TlGaS}_{2}$ single crystals has been investigated. The experimental results on high-frequency dielectric dispersion of dielectric coefficients of obtained $\mathrm{TlGaS}_{2}\langle 2 \mathrm{~mol} \% \mathrm{Ag}\rangle$ single crystals allowed to establish the nature of dielectric losses, the hopping mechanism of charge transport and to evaluate the density of localized states at the Fermi level, the average time of charge carrier hopping between localized states, average hopping length, scattering of trap states near the Fermi level and concentration of deep traps responsible for ac-conductivity. By Ag-doping of $\mathrm{TlGaS}_{2}$ single crystal the density of localized states at the Fermi level increased, but average hopping time and distance decreased.
\end{abstract}

